\section{AB0093 BLYS AND APRIL OVEREXPRESSION IN EARLY RHEUMATOID ARTHRITIS: ASSOCIATION WITH B CELLS AND MYELOID SUBSETS}

J. Rodríguez-Carrio ${ }^{1}$, M. Alperi-López ${ }^{2}$, P. López ${ }^{1}$, F.J. Ballina-García ${ }^{2}$ A. Suárez ${ }^{1} .{ }^{1}$ Area of Immunology, University of Oviedo; ${ }^{2}$ Department of Rheumatology, Hospital Universitario Central de Asturias, Oviedo, Spain

Background: studies on B cell-mediated autoimmune diseases highlight the relevance of the B Lymphocyte Stimulator (BLyS) and A Proliferation-Inducing Ligand (APRIL), but emerging evidence points to an interaction with cell lineages other than $B$ subsets. Although disturbances in the $B$ cell compartment underlie the early stages of rheumatoid arthritis (RA), this phenomenon is poorly understood. Objectives: to investigate the cellular populations responsible of BLyS expression and their association with the soluble forms of BLyS, APRIL and its receptor TACI (Transmembrane Activator and CALM Interactor) in early RA and to evaluate the changes in these parameters upon TNF $\alpha$-blockade.

Methods: membrane BLyS (mBLyS) expression was assessed on B cells, monocytes $(\mathrm{M} \varnothing)$, myeloid $(\mathrm{mDC})$ and plasmacytoid $(\mathrm{pDC})$ dendritic cells and neutrophils (NØ) by flow cytometry in fresh blood samples from 37 RA patients [DAS28 score (mean $\pm S D$ ): $4.84 \pm 1.44$, disease duration (mean (range)): 1.26 (0-11) years, $23(62.1 \%) \mathrm{RF}+, 19(51.3 \%) \mathrm{ACPA}+, 19$ untreated] and 31 healthy controls $(\mathrm{HC})$. A subgroup of 13 biologic-naïve RA patients was prospectively followed for three months upon TNF $\alpha$-blockade. Serum levels of soluble BLyS (sBLyS), APRIL (sAPRIL) and TACI (sTACl) were quantified by immunoassays. Results: mBLyS expression was increased on $B$ cells $(p=0.002), M \varnothing(p<0.001)$, mDC $(p<0.001)$ and NØ $(p=0.014)$ in RA patients. Higher sBLyS $(p=0.018)$ and sAPRIL $(p<0.001)$ serum levels were found in RA, whereas those of STACI were not different compared to HC $(p=0.460)$. Serum sAPRIL levels paralleled those of sTACI $(r=0.325, p=0.040)$, and mBLyS expression on $B$ cells $(r=0.463, p=0.009)$, $\mathrm{M} \varnothing(\mathrm{r}=0.521, \mathrm{p}=0.003), \mathrm{mDC}(\mathrm{r}=0.438, \mathrm{p}=0.014)$ and $\mathrm{N} \varnothing(\mathrm{r}=0.509, \mathrm{p}=0.009)$ in $\mathrm{HC}$ but not in RA patients. Serum levels of STACI were negatively associated with DAS28 score $(r=-0.272, p=0.006)$ in RA. However, sAPRIL was associated with $\mathrm{mBLyS}$ expression on $\mathrm{mDC}$ in patients with longer disease duration ( $>3$ months) $(r=0.779, p<0.001)$, but not in those recruited at onset $(r=0.245, p=0.361)$. In the whole RA group, TNF $\alpha$ serum levels were found to be correlated with SAPRIL $(r=0.499, p<0.001)$ and sBLyS $(r=0.362, p=0.013)$. Similarly, IFN $\alpha$ and SAPRIL were positively associated $(r=0.423, p<0.001)$. TNF $\alpha$-blockade was associated with decreasing $\mathrm{mBLyS}$ expression on $\mathrm{B}$ cells, $\mathrm{M} \varnothing, \mathrm{mDC}$ and $\mathrm{N} \varnothing$ (all $\mathrm{p}<0.050$ ) and a slight increase in sTACl $(p=0.064)$. Higher levels of mBLyS on $\mathrm{M} \varnothing$ and $\mathrm{mDC}$ at baseline were associated with a poor clinical response upon TNF $\alpha$-blockade ( $n=8 ; p=0.006$ and $p=0.010$ compared to $H C$, respectively).

Conclusions: a role for $\mathrm{B}$ cell-activating factors in the pathogenesis of early RA is supported. B cells and myeloid populations ( $M \varnothing, \mathrm{mDC}$ and $\mathrm{N} \varnothing)$ can account for the BLyS overexpression in RA, although important differences arise in their involvement. TNF $\alpha$ and IFN $\alpha$ are related to sBLyS and SAPRIL levels. An increased production of the soluble forms of BLyS and APRIL in addition to a less efficient feedback from their decoy receptors may delineate its detrimental effect. Disclosure of Interest: None declared

DOI: 10.1136/annrheumdis-2017-eular.2687

\section{AB0094 MIGRATION, COLONIZATION AND DISTRIBUTION OF BONE MARROW MESENCHYMAL STEM CELLS TRANSPLANTED IN CIA RATS WERE TRACED BY GREEN FLUORESCENT PROTEIN}

J. Hou, K. Xu, L. Zhang, G. Zhang, J. Gao, D. Ma, J. Fan. Shanxi Academy of Medical Sciences, Shan xi Da Yi Hospita, Taiyuan, China

Background: The mechanism of mesenchymal stem cells involved in injury and repair in vivo is not fully understood, so it is difficult to evaluate the effect of stem cells on rheumatoid arthritis.

Objectives: To investigate the migration, colonization and distribution of bone marrow mesenchymal stem cells (BM-MSCs) traced by green fluorescent protein (GFP) in the immune organs and joints of CIA rats, and study the mechanism of MSCs in repairing damage.

Methods: 1.MSCs labeled with GFP was cultured, amplified and identified in vitro.

2.Injected with mixture of II type collagen and complete Freund's adjuvant into Wistar rats space at 14 days: It was randomly divided into five groups, including early intervention group $(n=20)$, late intervention group $(n=20), C I A$ early control group $(n=12)$, late control group $(n=12)$ and normal control group $(n=12)$. (2) MSCs were injected from tail veins according to the number of $1 \times 10^{7} / \mathrm{kg}$, and the control groups were given equal volume of normal saline. (3) Observe the changes of arthritis index, arthritis swelling degree, and appearance of imageology and pathology.

3. The spleen, thymus, lymph nodes and joint tissues of the rats were made into pathological sections when transplanted at 3, 11, 30 and 42 days. The migration and distribution of the transplanted cells in the immune organs and the inflammatory joints were detected by immunohistochemistry.

Results: 1.(1)The arthritis index and degree of joint swelling in early and later intervention groups were decreased significantly than that of CIA control groups $(P<0.05)$. (2)The early intervention group had lower arthritis index and the degree of joint swelling than the later intervention group $(P<0.05)$.

2.The positive results of GFP was successfully detected by immunohistochemical method in the immune organs and joints of CIA rats, and sustainable for at least 42 days.

Conclusions: 1.MSCs transplanted through tail vein can migrate to the spleen, lymph nodes and thymus and joints, and can be long-term (42 days) colonization in these organizations.

2.The intervention of MSCs for CIA rats was effective, and early intervention effect was better than advanced intervention.

References:

[1] Gordon I. A. MacDonald, Andrea Augello, Cosimo De Bar. Role of mesenchymal stem cells in reestablishing immunologic tolerance in autoimmune rheumatic diseases. Arthritis \&Rheumatism. 2011 Spe, 63(9): 2547-557.

[2] David Guéit, Marie Maumus, Florence Apparailly, Christian Jorgensen \& Danièe Noë.Therapeutic mesenchymal stem or stromal cells in rheumatic diseases: rationale, clinicaldata and perspectivesClinical. Investigation. 2011 Sep, 1(9): 1269-1277.

Disclosure of Interest: None declared

DOI: 10.1136/annrheumdis-2017-eular.4018

\section{AB0095 BY INTRAVENOUS INFUSION MARKED BY GREEN FLUORESCENT PROTEIN TO REVEAL BONE MARROW MESENCHYMAL STEM CELLS' DISTRIBUTION AND DIFFERENTIATION OF COLLAGEN INDUCED ARTHRITIS RATS}

J. Gao, L. Zhang. Rheumatology Department, Shanxi Dayi Hospital, Taiyuan, China

Background: Rheumatoid arthritis (RA) is a autoimmune disease,which is characterized by the osteoclasia or the high deformity rate of cartilage and bone. According to some materials, Mesenchymal stem cells (MSCs) were definited as the cell full of proliferation, differentiation capacity, and potential immune regulation. MSCs transplantation could be a appropriate-designed pattern to the joint damaging from rheumatoid arthritis (RA).However, the repairing mechanism against osteoclasia of cartilage and bone is still unclear.

Objectives: This study used collagen induced arthritis (CIA) rats as animal model to explore MSCs' tissue repairing mechanism.

Methods: We observed the ability of BMSCs differentiating into cartilage cells by toluidine blue staining in vitro;Then,BMSCs were labeled by the green fluorescent protein (GFP), infused into CIA through rats' tail vein infusion. In different point time, the rat's joints were made paraffin section,we observed the differentation of GFP positive cells and the distribution of GFP-positive cells differentiated chondrocytes by immunohistochemicale method.

Results: First,we found BMSCs in vitro can differentiate into cartilage cells under a certain-culture condition. Then, BMSCs were labeled by the green fluorescent protein (GFP), infused into CIA through rats' tail vein infusion. In different point time, the rat's joints were made paraffin section, which the GFP positive cells were observed in synovium and bone marrow tissues after transplantation on the 3th day, and in cartilage tissues on the 11th day, then increased in cartilage tissues on the 30th day,42th day, by laser scanning confocal microscope. Anti-type II collagen, GFP double positive cells were found in articular cartilages (especially damaged part)by Anti-II collagen immunofluorescence technology.
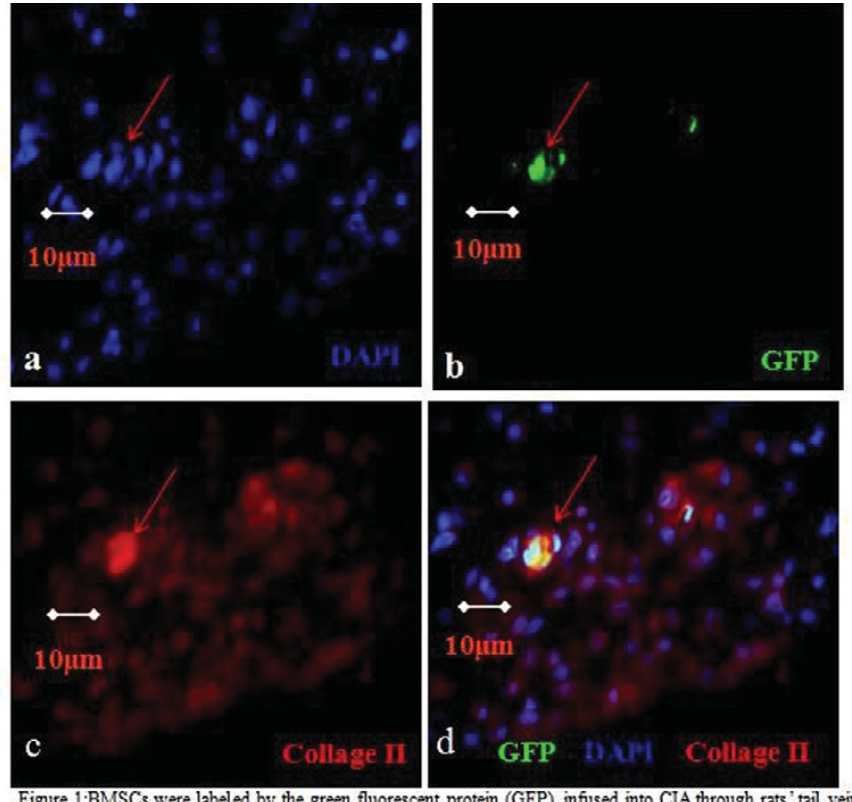

Figure 1:BMSCs were labeled by the green fluorescent protein (GFP), infused into CIA through rats 'tail vein infusion. Fluorescence microscopic observation of joint paraffin section. Chondrocyte were stained with an antibody specific to type II collagen and observed by fluorescence microscopy (red fluorescence). Nucles were stained with DAPI (blue fluorescence).GFP-MSCs were observed by fluorescence microscopy (green fluorescence).Anti-type II collagen and GFP double positive cells were found in articular cartilages. 